\title{
S100A2 coding sequence polymorphism: characterization and lack of association with psoriasis
}

\author{
Stefan W. Stoll,* Nicholas V. C. Chia, ${ }^{*}$ Rajan P. Nair,* Timothy L. Woods, * Philip Stuart, * \\ Tilo Henseler, + Stefan Jenisch, $\neq$ Enno Christophers, + John J. Voorhees* and James T. Elder* $, \S, \emptyset$
}

The Departments of *Dermatology and §Radiation Oncology, University of Michigan Medical School and $\mid$ Ann Arbor Veterans Affairs Hospital, Ann Arbor, MI, USA, and the Departments of +Dermatology and $¥$ Immunology, University of Kiel, Kiel, Germany

\begin{abstract}
Summary
Psoriasis is a chronic inflammatory skin disease with a strong genetic component. Linkage studies have identified several susceptibility loci for psoriasis including a region on chromosome 1q21 termed the 'epidermal differentiation complex'. At least 20 genes involved in epidermal differentiation and proliferation have been mapped to this region including S100A2, a gene known to be over-expressed in psoriasis lesions. In the course of cloning and sequencing several S100A2 cDNAs, we identified an A/G (Asn62Ser) polymorphism at nucleotide 185 of the S100A2 coding region. To determine whether this polymorphism is associated with psoriasis, we tested DNA from 38 unrelated normal and 40 unrelated psoriatic individuals. The $185 \mathrm{G}$ allele was present in 148 of the 156 chromosomes analysed, giving an allele frequency of $94.9 \%$. All of the remaining chromosomes carried 185A. There was no significant difference in the allele distribution between normal and psoriatic individuals (normals 72G, 4A; psoriatics 76G, 4A; $P=1.00$ by Fisher's exact test). Our data explain conflicting results in the literature regarding the sequence of S100A2 but provide no support for a direct causal role for S100A2 in psoriasis.
\end{abstract}

\section{Introduction}

Psoriasis is a chronic inflammatory skin disease associated with increased proliferation and altered differentiation of keratinocytes, in the context of inappropriate activity of antigen presenting cells and T lymphocytes. ${ }^{1}$ Multiple lines of evidence suggest that psoriasis is a polygenic disease, ${ }^{2}$ and several candidate loci have emerged from recent linkage studies. ${ }^{3}$ One of these loci maps to human chromosomal band $1 \mathrm{q} 21,{ }^{4}$ home of the epidermal differentiation complex (EDC) - a region containing at least 20 genes involved in epidermal differentiation. ${ }^{5}$

S100A2 belongs to the expanding family of S100 proteins. $^{6}$ Of the 16 known members of the human

Correspondence: J. T. Elder, 3312 CCGC Box 0932, University of Michigan, Ann Arbor, Ml 48109-0932, USA. Tel.: +1 7347630355.

Fax: +1 734763 4575. E-mail: jelder@umich.edu

Accepted for publication 6 October 2000
S100 gene family, 13 are clustered in the EDC. Structurally related to calmodulin, S100 proteins share two conserved calcium-binding domains of the EF-hand type that are separated by a more variable hinge region. While many functions have been proposed for S100 proteins, their true function remains enigmatic. Originally named CaN19 when it was identified in a screen for tumour suppressor genes, ${ }^{7}$ sequence comparisons revealed that $S 100 \mathrm{~A} 2$ is the human homologue of bovine S100L. ${ }^{8}$ CaN19 was renamed S100A2 based on its relatively telomeric position in the S100A gene cluster. ${ }^{9}$

Several lines of evidence suggest S100A2 as a candidate gene for familial psoriasis. In addition to its location in the EDC, we have previously shown that S100A2 mRNA is over-expressed in hyperproliferative keratinocytes of psoriasis lesions and peri-tumoral skin. ${ }^{10,} 11$ Psoriasis and wound healing are characterized by increased signal transduction through the c-ErbB family of receptor tyrosine kinases, ${ }^{12}$ and we 
have recently shown that transcription of S100A2 is strongly up-regulated in keratinocytes after c-ErbB activation. $^{13}$

In the course of cloning and sequencing several S100A2 cDNAs, we have identified a sequence polymorphism 185A/G within the S100A2 coding region (this numbering corresponds to position 222 of Genbank ID M87068). This mutation results in an amino acid change near the C-terminal, high-affinity EF-hand of S100A2 (Asn62Ser). To confirm this polymorphism, and to determine whether it plays an aetiologic role in psoriasis, we analysed the S100A2 allele distribution in DNA from 38 normal and 40 psoriatic individuals using a recently developed mutation detection assay. ${ }^{14,15}$

\section{Subjects and methods}

DNA was prepared from the peripheral blood of 78 unrelated individuals as described previously. ${ }^{16}$ Informed consent was obtained from all subjects according to the guidelines of the Institutional Review Board of the University of Michigan Medical School. Forty unrelated individuals were selected from our ongoing study of familial psoriasis, ${ }^{16,17}$ based on a personal history of juvenile onset chronic plaque type psoriasis (age of onset $<40$ years) and the presence of psoriasis in at least one first-degree relative. This group consisted of 19 males and 21 females with present ages from 5 to 68 years (mean, 41.3 years). Thirty-eight unrelated normal volunteers were used as control subjects (19 females and 19 males; age range, 1286 years, mean age, 54.3 years). Among the 78 individuals recruited for the study, 19 individuals were recruited in Kiel, Germany (10 psoriatic and nine normal individuals). All other individuals were recruited in Michigan (30 psoriatic and 29 normal individuals). All individuals were evaluated by dermatologists for the presence or absence of psoriasis. None of the controls had a personal or family history of psoriasis.

S100A2 clones were isolated by screening a human keratinocyte library (Clontech, HL45) or by reverse transcription (RT)-polymerase chain reation (PCR) amplification of total RNA isolated from normal human keratinocytes as described. ${ }^{13,} 18$ Inserts of positive bacteriophage clones were isolated and cloned into pUC19 and sequenced according to the method of Sanger ${ }^{19}$ either in the laboratory or through the DNA Sequencing Core of the University of Michigan. Alternatively, PCR fragments were gel-purified and cloned into pCRII $^{\mathrm{TM}}$ (Invitrogen, Carlsbad, CA, USA) and subjected to DNA sequencing by the Sanger method.
The frequency of the S100A2 185A/G nucleotide polymorphism was determined by using base excision sequence scanning analysis $\left(\right.$ BESS $^{\mathrm{TM}}$, Epicentre, Madison, WI, USA). ${ }^{14,}{ }^{15}$ A fragment of genomic DNA incorporating the S100A2 $185 \mathrm{~A} / \mathrm{G}$ polymorphism was amplified by using forward primer 5'-TGTGTGAGGGTCTGGGTGTTTG-3' (in intron 2) and reverse primer 5'-AAGAGTTCTGCTTCAGGGTCGG-3' (in exon 3, Genbank ID Y07755). For the BESS-T ${ }^{\mathrm{TM}}$ scan, $50 \mathrm{ng}$ of genomic DNA was subjected to PCR $\left(30\right.$ cycles of: $94^{\circ} \mathrm{C}$, $45 \mathrm{~s} ; 55^{\circ} \mathrm{C}, 45 \mathrm{~s} ; 72{ }^{\circ} \mathrm{C}, 45 \mathrm{~s}$ ) in a final volume of $25 \mu \mathrm{L}$ using BESS dNTP mix and $\left[\gamma^{-32} \mathrm{P}\right]$ ATP endlabelled reverse primer. Amplification mix $(5 \mu \mathrm{L})$ was incubated with $1 \mu \mathrm{L}$ BESS-T ${ }^{\mathrm{TM}}$ excision enzyme mix for $30 \mathrm{~min}$ at $37^{\circ} \mathrm{C}$ to randomly cleave incorporated dUMP residues. For the BESS-G ${ }^{\mathrm{TM}}$ scan, PCR products were amplified with BESS-dNTP mix in the presence of $\left[\gamma^{32} \mathrm{P}\right]$ ATP end-labelled forward primer. To specifically modify guanine bases on amplified DNA, $5 \mu \mathrm{L}$ of the PCR reaction were mixed with $1 \mu \mathrm{L}$ BESS-G ${ }^{\mathrm{TM}}$ modification reagent and exposed for $15 \mathrm{~min}$ to visible light on a light box, vortexing the mixture every $5 \mathrm{~min}$. After addition of $1 \mu \mathrm{L}$ of BESS- $\mathrm{G}^{\mathrm{TM}}$ excision mix, the reaction was incubated for $30 \mathrm{~min}$ at $37^{\circ} \mathrm{C}$ to randomly cleave DNA strands on modified guanine bases. Both excision reactions were stopped by addition of $5 \mu \mathrm{L}$ of stop solution, denatured $\left(90{ }^{\circ} \mathrm{C}, 10 \mathrm{~min}\right)$ and electrophoresed on an $8 \%$ urea-polyacrylamide sequencing gel. The radioactive fragments were identified by autoradiography. To confirm the results obtained by base excision sequence scanning analysis, PCR fragments obtained from selected samples were gel purified and sequenced as described above.

Allele frequencies in normal and psoriatic individuals were compared using Fisher's exact test of independence. Power calculations were performed using a Monte Carlo method. The calculations assumed a disease prevalence of 0.02 and a population frequency of 0.0513 for the A allele (8/156 occurrences; cases and controls were grouped together). The number of $\mathrm{A}$ alleles in the case and control samples was based on the estimated frequencies of the A allele in the two populations, the estimated variance (which is a function of frequency and sample size), and sample sizes of 80 and 76 chromosomes for cases and controls, respectively. The ratio of A allele frequency in case vs. control chromosomes was an adjustable parameter used to represent the strength of the presumed disease association. The null hypothesis of independence of A allele frequency and disease phenotype was assessed with Fisher's exact test, and a nominal two-tailed $P$-value of 0.05 was the threshold 
for declaring significance. Power was defined as the fraction of 10000 tests that were significant.

\section{Results}

One of the S100A2 cDNA clones sequenced contained an $\mathrm{A}$ at nucleotide 185 in the coding region, whereas the remaining cDNAs contained a $\mathrm{G}$ at this position. This polymorphism results in an amino acid change from asparagine to serine (Asn62Ser) of the S100A2 protein sequence. No other polymorphisms were detected in a total of four independent S100A2 cDNAs subjected to sequencing.

Base excision sequence scanning analysis revealed that 148 out of the 156 chromosomes tested carried the $\mathrm{G}$ allele, yielding an allele frequency of $94.9 \%$. The remaining eight chromosomes carried the A allele. Seventy-two G and four A alleles were observed in the normal group, as compared to $76 \mathrm{G}$ and four A alleles in the psoriatic group. The results obtained by base excision sequence scanning analysis were confirmed by sequencing a select set of samples (Fig. 1). Among the 78 individuals involved in the study one individual, a 47-year-old male belonging to the normal control group, was homozygous for the A allele (Fig. 1).

There was no significant difference in the allele frequency between the two groups ( $P=1.00$ by Fisher's exact test, two-tailed). Monte Carlo simulations revealed that our sample had $50 \%$ power to detect a significant association at the $P=0.05$ level if the polymorphism carried a relative risk of $3,79 \%$ power if the polymorphism carried a relative risk of 4 , and $94 \%$ power if the polymorphism carried a relative risk of 5 .

\section{Discussion}

Heizmann and $\mathrm{Cox}^{6}$ commented that their sequencing results for S100A2 were at variance with an earlier report of Lee et al. ${ }^{7}$ because Heizmann et al. found G instead of $\mathrm{A}$ at nucleotide 185 of the coding sequence in cDNA, and because the same variant was found in genomic DNA. We identified both sequences in the course of cloning several S100A2 cDNAs, providing suggestive evidence for a S100A2 gene polymorphism. To confirm this polymorphism, and to determine whether it might play a role in the genetics of psoriasis, we analysed a sample of 40 juvenile onset psoriatics and 38 sex- and age-matched controls. We found that the A allele was rare, being found on only eight of 156 chromosomes. The relative rarity of the A allele probably accounts for the interpretation of the available cDNA data as indicative of a sequencing error.

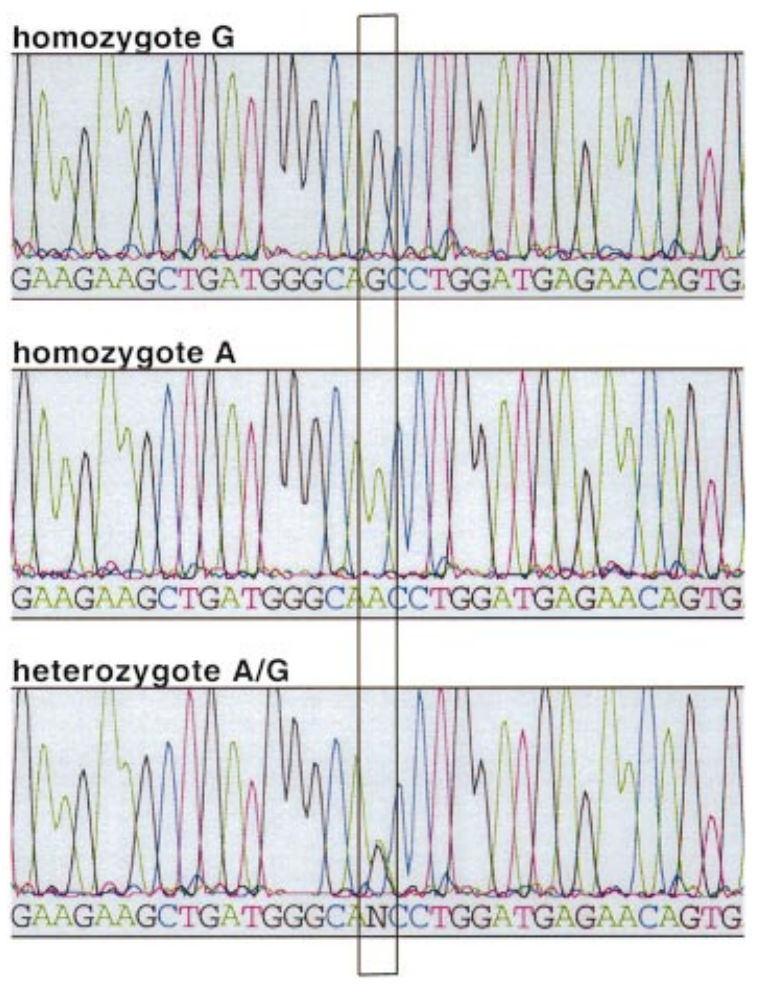

Figure 1 Sequence of S100A2 surrounding the 185A/G polymorphism. Automated sequencer outputs are shown. The rectangles highlight the polymorphism. The sequences shown were derived from sequencing of gel-purified PCR products prepared from genomic DNA using the primers described. The sequence derived from PCR-amplified DNA of a heterozygous individual shows two overlapping peaks ( $\mathrm{G}$ and $\mathrm{A}$ ) at this position and is indicated by an $\mathrm{N}$ in the sequence.

We were unable to identify any significant association of the polymorphism with psoriasis in a sample of 38 normal and 40 psoriatic individuals. Our power calculations indicate that we are unlikely to have missed a locus of major effect. However, we cannot rule out an involvement of this gene if it is causal in only a subset of individuals, or if the magnitude of its effect is small. In any case, the absolute number of occurrences of the $\mathrm{A}$ allele was as low in the psoriatic group as it was in the normal group (four out of 80 alleles and four out of 76 alleles, respectively). Moreover, the only individual identified by the study as homozygous for the A allele was a 47-year-old male belonging to the normal controls. These observations provide additional support for the assertion that the 185A allele of S100A2 does not play a significant role in the genetics of psoriasis.

These results are consistent with linkage studies from our laboratory, which have not provided significant evidence for a psoriasis susceptibility locus on chromosome 1q21, despite some evidence for segregation of 
1q21 markers in certain families (Nair et al., ${ }^{17}$ and unpublished data). However, evidence for a 1q21 locus has been reported by at least two laboratories. ${ }^{3,4}$ Failure to replicate a linkage finding is neither uncommon nor unexpected in the complex genetic disorders. ${ }^{20}$ The subjects in our study were deliberately chosen without reference to the 1q21 linkage status of their families. We cannot exclude the possibility that a positive result would have been obtained if the study were much larger or if more individuals belonging to 1q21-linked families had been analysed. It also remains possible that an EDC gene other than S100A2 could play a causative role in psoriasis. Recently, the coding and regulatory regions of the S100A7 (psoriasin) gene were screened for possible disease-associated variations. ${ }^{21}$ However, that study could identify no disease-related sequence variations in a total of 80 chromosomes evaluated in that study (50 normal, 30 psoriatic). As allelic variation of the human genome becomes better characterized in terms of singlenucleotide polymorphisms, ${ }^{22}$ the availability of welldefined clinical collections will allow us to determine whether any of the remaining EDC genes play a causal role in psoriasis.

\section{Acknowledgements}

This work was supported by the Veterans Affairs Merit Review (J. T. E.), by a grant (R01 AR4274) from the National Institute of Arthritis, Musculoskeletal and Skin Diseases, NIH, by an award (DFG-WE 905/1-1) from the German Research Foundation (T. H., S. J., E. C.), by a Chesebrough/Lever Brothers Dermatology Foundation Career Development Award (S. W. S.), and by the Babcock Memorial Trust. Dr Elder is supported by the Ann Arbor Veterans Affairs Hospital. Portions of these studies were conducted at the General Clinical Research Center at the University of Michigan, funded by a grant (M01EE00042) from the National Center for Research Resources, National Institutes of Health, USPHS.

\section{References}

1 Griffiths CE, Voorhees JJ. Psoriasis, T cells and autoimmunity. J R Soc Med 1996; 89 (6): 315-9.

2 Elder JT, Nair RP, Guo SW, Henseler T, Christophers E, Voorhees JJ. The genetics of psoriasis. Arch Dermatol 1994; 130 (2): 216-24.

3 Bhalerao J, Bowcock AM. The genetics of psoriasis: a complex disorder of the skin and immune system. Hum Mol Genet 1998; 7 (10): 1537-45.

4 Capon F, Novelli G, Semprini S et al. Searching for psoriasis susceptibility genes in Italy: genome scan and evidence for a new locus on chromosome 1. J Invest Dermatol 1998; 112 (1): $32-5$.

5 South AP, Cabral A, Ives JH et al. Human epidermal differentiation complex in a single $2.5 \mathrm{mbp}$ long continuum of overlapping DNA cloned in bacteria integrating physical and transcript maps [in process citation]. J Invest Dermatol 1999; 112 (6): 910-8.

6 Heizmann CW, Cox JA. New perspectives on S100 proteins: a multi-functional $\mathrm{Ca}(2+)-$, Zn (2+)- and $\mathrm{Cu}(2+)-$ binding protein family. Biometals 1998; 11 (4): 38397.

7 Lee SW, Tomasetto C, Swisshelm K, Keyomarsi K, Sager R. Down-regulation of a member of the $S 100$ gene family in mammary carcinoma cells and reexpression by azadeoxycytidine treatment. Proc Natl Acad Sci USA 1992; 89 (6): 2504-8.

8 Glenney J Jr, Kindy MS, Zokas L. Isolation of a new member of the S100 protein family: amino acid sequence, tissue, and subcellular distribution. J Cell Biol 1989; 108 (2): 569-78.

9 Schafer BW, Wicki R, Engelkamp D, Mattei MG, Heizmann CW. Isolation of a YAC clone covering a cluster of nine S100 genes on human chromosome 1q21: rationale for a new nomenclature of the S100 calcium-binding protein family. Genomics 1995; 25 (3): 638-43.

10 Hardas BD, Zhao X, Zhang J, Longqing X, Stoll S, Elder JT. Assignment of psoriasin to human chromosomal band 1q21: Coordinate overexpression of clustered genes in psoriasis. J Invest Dermatol 1996; 106 (4): 753-8.

11 Xia L, Stoll SW, Liebert M et al. CaN19 expression in benign and malignant hyperplasias of the skin and oral mucosa: evidence for a role in regenerative differentiation. Cancer Res 1997; 57 (14): 3055-62.

12 Stoll S, Garner W, Elder J. Heparin-binding ligands mediate autocrine EGF receptor activation in skin organ culture. J Clin Invest 1997; 100 (5): 1271-81.

13 Stoll SW, Zhao X, Elder JT. EGF stimulates transcription of CaN 19 (S100A2) in HaCaT keratinocytes. J Invest Dermatol 1998; 111 (6): 1092-7.

14 Charrel RN, Levy N, Tesh RB, Chandler LJ. Use of base excision sequence scanning for detection of genetic variations in St. Louis encephalitis virus isolates. J Clin Microbiol 1999; 37 (6): 1935-40.

15 Brieger J, Weidt EJ, Gansen K, Decker HJ. Detection of a novel germline mutation in the von Hippel-Lindau tumoursuppressor gene by fluorescence-labelled base excision sequence scanning (F-BESS). Clin Genet 1999; 56 (3): 210-5.

16 Nair R, Guo S, Jenisch S et al. Scanning chromosome 17 for psoriasis susceptibility: lack of evidence for a distal $17 \mathrm{q}$ locus. Hum Hered 1995; 45: 219-30.

17 Nair RP, Henseler T, Jenisch S et al. Evidence for two psoriasis susceptibility loci (HLA and 17q) and two novel candidate regions (16q and 20p) by genome-wide scan. Hum Mol Genet 1997; 6 (8): 1349-56.

18 Zhao XP, Elder JT. Positional cloning of skin-specific genes 
from the human epidermal differentiation complex. Genomics 1997; 45: 250-8.

19 Sanger F, Nicklen S, Coulson AR. DNA sequencing with chain-terminating inhibitors. Proc Natl Acad Sci USA 1977; 74 (12): 5463-7.

20 Lander E, Kruglyak L. Genetic dissection of complex traits: guidelines for interpreting and reporting linkage results. Nature Genet 1995; 11 (3): 241-7.
21 Semprini S, Capon F, Bovolenta S et al. Genomic structure, promoter characterisation and mutational analysis of the S100A7 gene: exclusion of a candidate for familial psoriasis susceptibility. Hum Genet 1999; 104 (2): 130-4.

22 Buetow KH, Edmonson MN, Cassidy AB. Reliable identification of large numbers of candidate SNPs from public EST data. Nature Genet 1999; 21 (3): 323-5. 